\title{
Influence of Crystal Packing on the
}

\section{Resonant Enhancement of the Second-Order}

\section{Nonlinear Optical Response of Dipolar Chromophores}

\author{
Wei Zhang, Anthony F. Cozzolino, Amir H. Mahmoudkhani, Mark Tulumello, Sarah Mansour, \\ and Ignacio Vargas-Baca.*
}

Department of Chemistry, McMaster University, 1280 Main Street West, Hamilton, Ontario L8S 4M1.

vargas@ chemistry.mcmaster.ca

Supporting Information 


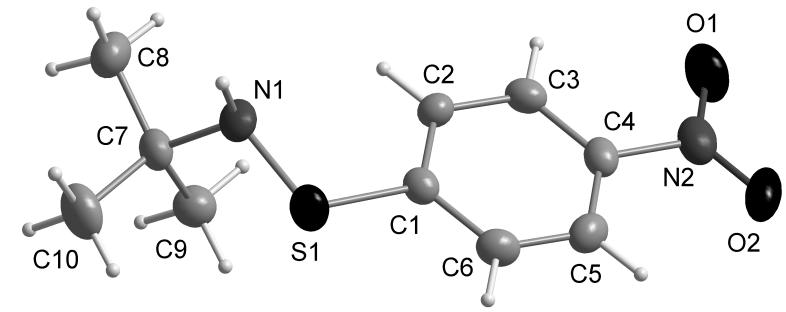

Figure S1. ORTEP diagram of the asymmetric unit in the crystal structure of 2.

$\underline{\text { Table S1. Crystal data and structure refinement for } \mathbf{2}}$.
Empirical formula
C10 H14 N2 O2 S

CCDC Number: 261885

Crystal system, space group a $[\AA]$

$\mathrm{b}[\AA]$

c $[\AA]$

Volume $\left[\AA^{3}\right]$

$\mathrm{Z}, \rho$ (calc.) $\left[\mathrm{g} . \mathrm{cm}^{-3}\right]$

$\mathrm{T}[\mathrm{K}]$

$\mu\left[\mathrm{mm}^{-1}\right]$

$\theta$ range $\left[{ }^{\circ}\right]$

Limiting indices

Orthorhombic, Fdd2 (No. 43)

24.370(9)

$31.908(11)$

$5.941(2)$

4620(3)

$16,1.301$

173(2)

0.263

$2.10-27.47$

$-31<=\mathrm{h}<=30$

$-41<=\mathrm{k}<=41$

$-7<=1<=7$

Reflections collected / unique

R(int.)

$10219 / 2225$

0.042

Parameters

156

$\mathrm{R} 1 * / \mathrm{wR} 2 *(\mathrm{I}>2 \sigma(\mathrm{I}))$

$0.035 / 0.079$

$\mathrm{R} 1 * / \mathrm{wR} 2 *$ for all data

Goodness-of-fit on $\mathrm{F}^{2}$

$0.052 / 0.086$

1.024

Larg. diff. peak/ hole [e. $\left.\AA^{-3}\right]$

$0.196 /-0.212$

$* \mathrm{R} 1=\Sigma\left\|\mathrm{F}_{\mathrm{o}}|-| \mathrm{F}_{\mathrm{c}}\right\| / \Sigma\left|\mathrm{F}_{\mathrm{o}}\right|, \mathrm{wR} 2=\left\{\Sigma\left[\mathrm{w}\left(\mathrm{F}_{\mathrm{o}}{ }^{2}-\mathrm{F}_{\mathrm{c}}{ }^{2}\right)^{2}\right] / \Sigma \mathrm{w}\left(\mathrm{F}_{\mathrm{o}}{ }^{2}\right)^{2}\right\}^{1 / 2}$ 
Table S2. Selected bond lengths $[\AA ̊]$, bond angles $\left[^{\circ}\right]$ and torsion angles $\left[^{\circ}\right]$ for $\mathbf{2}$.

$\begin{array}{lllr}\mathrm{S}(1)-\mathrm{N}(1) & 1.684(2) & \mathrm{C}(5)-\mathrm{C}(4)-\mathrm{N}(2) & 119.0(2) \\ \mathrm{S}(1)-\mathrm{C}(1) & 1.763(2) & \mathrm{O}(2)-\mathrm{N}(2)-\mathrm{O}(1) & 123.4(2) \\ \mathrm{C}(7)-\mathrm{N}(1) & 1.487(3) & \mathrm{O}(2)-\mathrm{N}(2)-\mathrm{C}(4) & 118.6(2) \\ \mathrm{N}(1)-\mathrm{S}(1)-\mathrm{C}(1) & 102.4(1) & \mathrm{O}(1)-\mathrm{N}(2)-\mathrm{C}(4) & 118.0(2) \\ \mathrm{C}(2)-\mathrm{C}(1)-\mathrm{S}(1) & 123.2(2) & \mathrm{C}(7)-\mathrm{N}(1)-\mathrm{S}(1) & 118.9(2) \\ \mathrm{C}(6)-\mathrm{C}(1)-\mathrm{S}(1) & 116.9(2) & \mathrm{C}(7)-\mathrm{N}(1)-\mathrm{H}(1) & 113.3(19) \\ \mathrm{N}(1)-\mathrm{C}(7)-\mathrm{C}(9) & 109.5(2) & \mathrm{S}(1)-\mathrm{N}(1)-\mathrm{H}(1) & 108.9(19) \\ \mathrm{N}(1)-\mathrm{C}(7)-\mathrm{C}(8) & 105.8(2) & & \\ \mathrm{N}(1)-\mathrm{C}(7)-\mathrm{C}(10) & 111.8(2) & \mathrm{N}(1)-\mathrm{S}(1)-\mathrm{C}(1)-\mathrm{C}(2) & 1.1(2) \\ \mathrm{C}(3)-\mathrm{C}(4)-\mathrm{N}(2) & 119.1(2) & \mathrm{O}(2)-\mathrm{N}(2)-\mathrm{C}(4)-\mathrm{C}(5) & -0.1(3) \\ \mathrm{O}(2)-\mathrm{N}(2) & 1.222(3) & \mathrm{O}(1)-\mathrm{N}(2)-\mathrm{C}(4)-\mathrm{C}(5) & 179.5(2) \\ \mathrm{C}(4)-\mathrm{N}(2) & 1.462(3) & \mathrm{O}(1)-\mathrm{N}(2)-\mathrm{C}(4)-\mathrm{C}(3) & -1.1(3) \\ \mathrm{N}(2)-\mathrm{O}(1) & 1.235(3) & \end{array}$

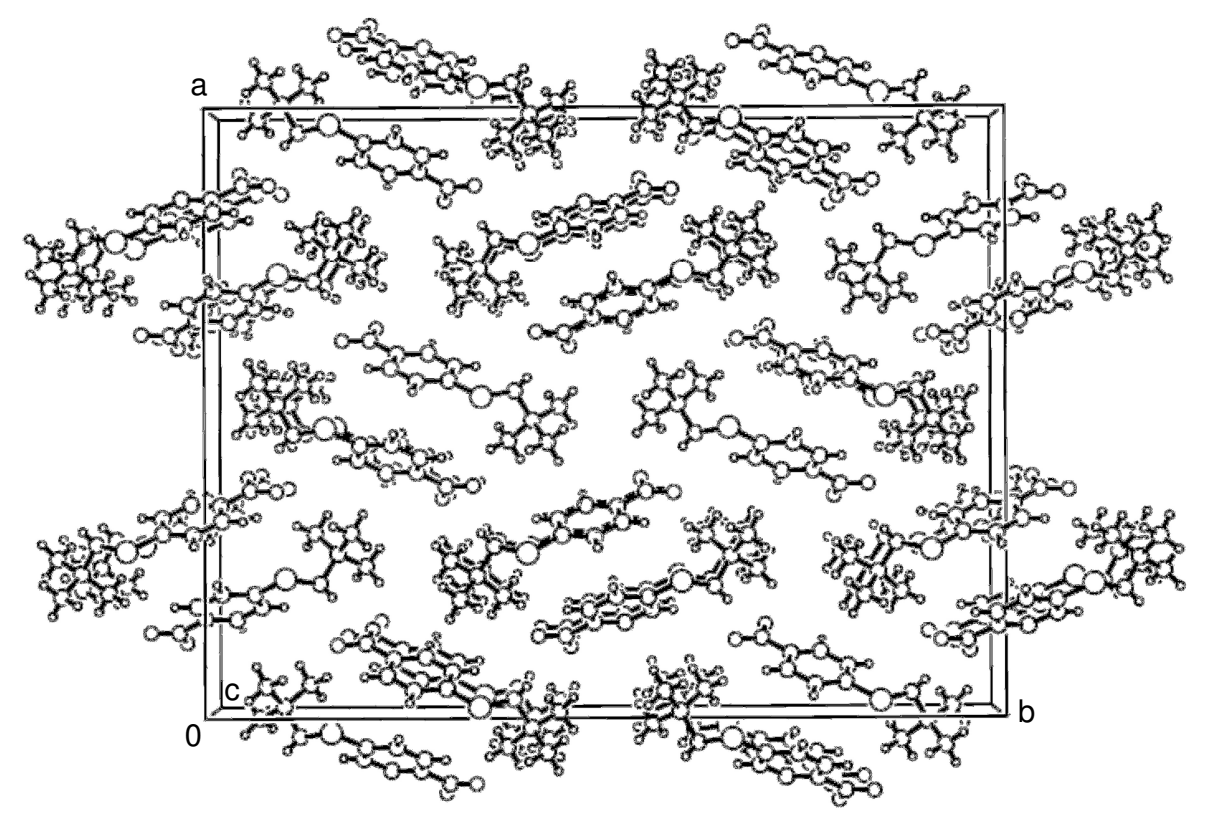

Figure S2. Packing diagram of 2 viewed along the c axis. 
Table S3. Hydrogen-bond dimensions for $2\left[{ }^{\mathrm{A}},{ }^{\circ}\right]$.

$\begin{array}{lrrrr}\text { D-H...A } & \text { d(D-H) } & \mathrm{d}(\mathrm{H} \ldots \mathrm{A}) & \mathrm{d}(\mathrm{D} \ldots \mathrm{A}) & <(\mathrm{DHA}) \\ \mathrm{N}(1)-\mathrm{H}(1) \ldots \mathrm{O}(1)^{\mathrm{a}} & 0.80(3) & 2.37(3) & 3.170(3) & 171(3) \\ & & & \end{array}$

Table S4. Calculated electronic transitions of $\mathbf{1}$, its dimer $\mathbf{1}_{2}$, and $\mathbf{2}$.

\begin{tabular}{rccccccccc} 
Transition & \multicolumn{1}{c}{$\mathbf{1}$} & \multicolumn{9}{c}{$\mathbf{1}_{2}$} & \multicolumn{3}{c}{$\mathbf{2}$} \\
\hline & $\mathrm{eV}$ & $\mathrm{nm}$ & $\mathrm{f}$ & $\mathrm{eV}$ & $\mathrm{nm}$ & $\mathrm{f}$ & $\mathrm{eV}$ & $\mathrm{nm}$ & $\mathrm{f}$ \\
1 & 3.3 & 379 & 0.3822 & 2.4 & 520 & 0.0021 & 3.0 & 420 & 0.0385 \\
2 & 3.7 & 333 & 0.0000 & 2.7 & 452 & 0.0031 & 3.1 & 403 & 0.3054 \\
3 & 3.8 & 326 & 0.0048 & 3.3 & 381 & 0.0015 & 3.7 & 334 & 0.0057 \\
4 & 4.2 & 297 & 0.0002 & 3.3 & 380 & 0.0004 & 3.8 & 328 & 0.0002 \\
5 & 4.4 & 280 & 0.0299 & 3.4 & 361 & 0.5504 & 4.2 & 296 & 0.0001 \\
6 & 5.0 & 246 & 0.0614 & 3.6 & 346 & 0.0540 & 4.3 & 291 & 0.0091 \\
7 & 5.1 & 243 & 0.0002 & 3.6 & 341 & 0.0019 & 4.7 & 267 & 0.0001 \\
8 & 5.3 & 235 & 0.0061 & 3.7 & 338 & 0.0125 & 4.7 & 261 & 0.1202 \\
9 & 5.4 & 231 & 0.0002 & 3.7 & 338 & 0.0102 & 4.9 & 251 & 0.0016 \\
10 & 5.5 & 226 & 0.0004 & 3.8 & 327 & 0.0001 & 5.0 & 250 & 0.0144 \\
11 & 5.5 & 225 & 0.0013 & 3.9 & 317 & 0.0011 & 5.2 & 241 & 0.0000 \\
12 & 5.5 & 223 & 0.0001 & 4.0 & 308 & 0.0047 & 5.2 & 239 & 0.0473 \\
13 & 5.6 & 222 & 0.0001 & 4.0 & 307 & 0.0055 & 5.3 & 235 & 0.0001 \\
14 & 5.6 & 220 & 0.0038 & 4.1 & 305 & 0.0009 & 5.4 & 232 & 0.0099 \\
15 & 5.7 & 217 & 0.0761 & 4.1 & 302 & 0.0006 & 5.5 & 227 & 0.0002 \\
16 & 5.7 & 217 & 0.0323 & 4.1 & 299 & 0.0003 & 5.6 & 220 & 0.0014 \\
17 & 5.9 & 211 & 0.0088 & 4.2 & 296 & 0.0002 & 5.7 & 217 & 0.0010 \\
18 & 5.9 & 209 & 0.0513 & 4.2 & 294 & 0.0000 & 5.7 & 217 & 0.0270 \\
19 & 6.1 & 204 & 0.0029 & 4.3 & 287 & 0.0020 & 5.7 & 216 & 0.0248 \\
20 & 6.1 & 204 & 0.1040 & 4.5 & 276 & 0.0014 & 5.8 & 214 & 0.0008 \\
\hline
\end{tabular}

